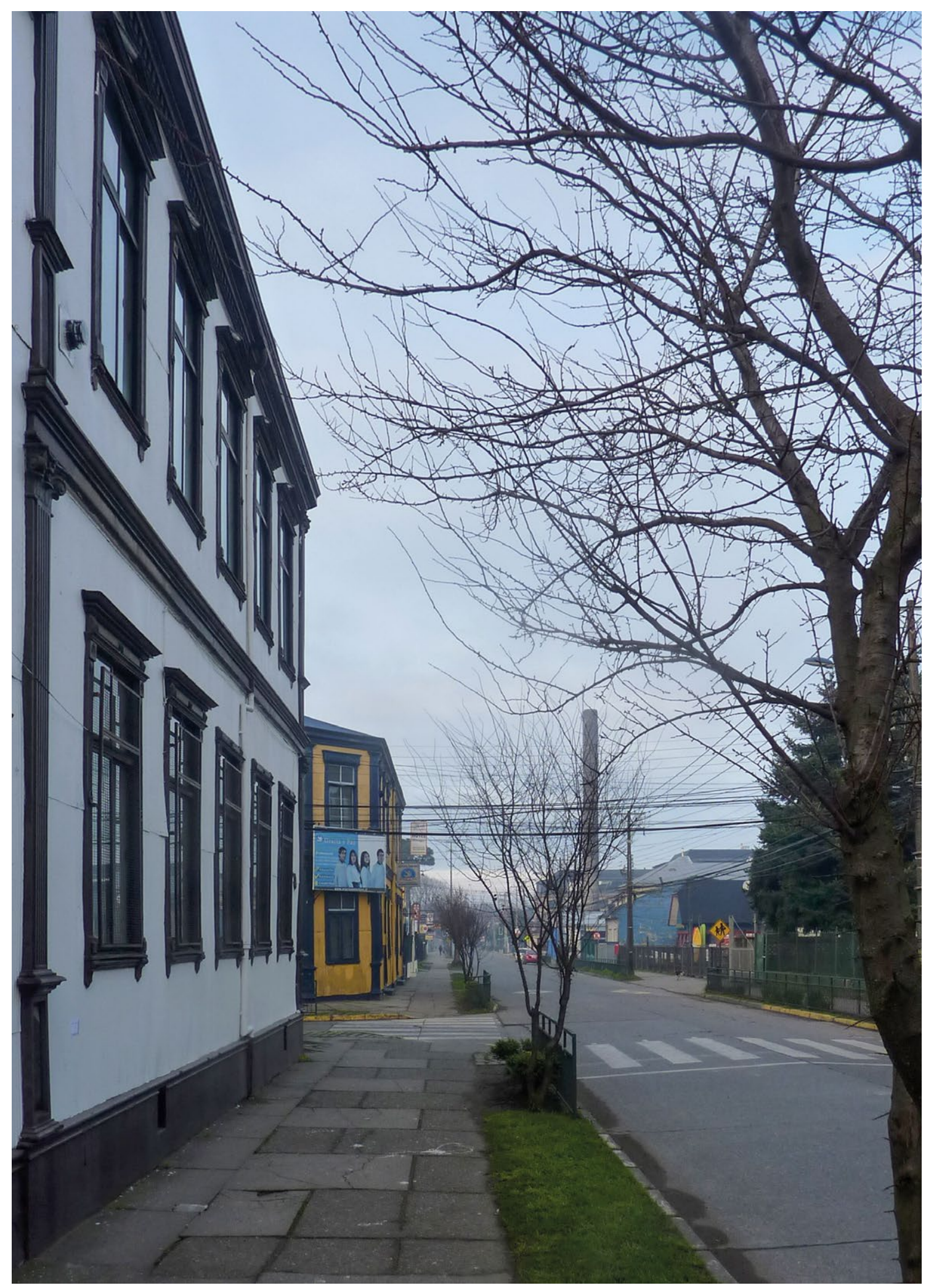

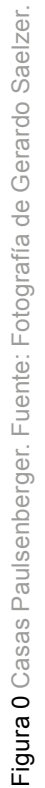



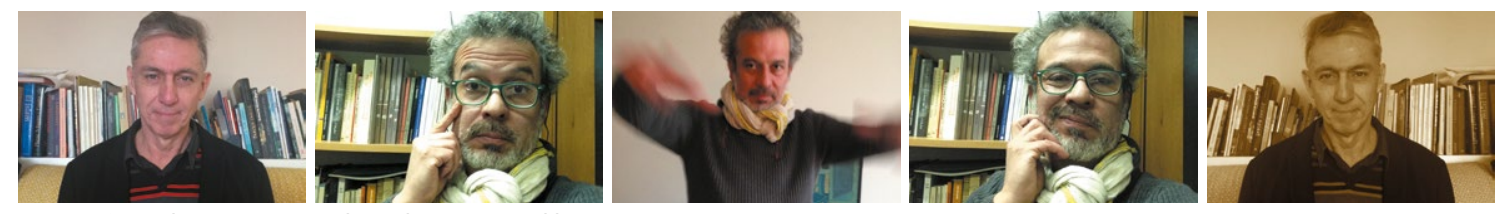

Fotos: Pedro Araya y Gerardo Saelze

\title{
RESTAURACIÓN E IMAGINARIO URBANO: ALGUNAS OBSERVACIONES ANTROPOLÓGICO-ARQUITECTÓNICAS EN TORNO A LA IDEA DE PATRIMONIO'
}

\author{
RESTORATION AND URBAN IMAGINARY: SOME ANTHROPOLOGICAL-ARCHITECTURAL \\ OBSERVATIONS ON THE IDEA OF HERITAGE'
}

Pedro Araya Riquelme², Gerardo Saelzer Canouet ${ }^{3}$

RESUMEN

El presente artículo propone algunas observaciones a partir del diálogo antropológico con el pensar y el hacer arquitectónico, en el marco de la intervención/restauración de un inmueble de valor histórico: la casa Ehrenfeld, actual Conservatorio de Música de la Universidad Austral de Chile (UACh), ubicada en la ciudad de Valdivia. Considerando que los inmuebles conocen una heterogeneidad de materiales, de acciones y de actores, se pretende apuntar a una serie de procesos, tanto arquitectónicos como sociales, que develan otros ecos en el espacio urbano y que inducen a ampliar la mirada más allá de una determinada construcción y su contexto patrimonial inmediato. De tal forma, se perciben múltiples apropiaciones materiales que abren nuevos espacios de interpretación respecto a las nociones de patrimonio y de tipologías urbanas.

Palabras clave: casas históricas, restauración, barrio, tipología, dinámicas sociales.

\section{ABSTRACT}

This article proposes some observations that stem from an anthropological point of view with an architectural way of thinking and doing, as they emerge when intervening in/restoring a building of historical value: the Ehrenfeld house, at present the Universidad Austral de Chile's (UACh) Conservatory of Music, located in the city of Valdivia. Considering that buildings experience heterogeneous materials, actions and actors, a series of processes, both architectural and social, are highlighted that reveal other echoes in urban space and lead us to broaden our perspective beyond the given building and its immediate heritage context. Thus, a number of material appropriations are discerned that open new spaces of interpretation concerning notions of heritage and urban typologies.

Keywords: historical houses, restoration, neighborhood, typology, social dynamics.

Artículo recibido el 13 de diciembre de 2016 y aceptado el 11 de junio de 2017 DOI: https://doi.org/10.22320/07196466.2017.35.051.03

[1] Este artículo está basado en los resultados de investigación generados en el proyecto financiado por el Consejo Nacional de la

Cultura y las Artes, Fondo del Patrimonio Cultural, convocatoria 2013, y la Universidad Austral de Chile.

[2] Instituto de Estudios Antropológicos, Universidad Austral de Chile, Valdivia, Chile. pedro.araya@uach.c

[3] Instituto de Arquitectura y Urbanismo, Universidad Austral de Chile, Valdivia, Chile. gerardo.saelzer@uach.cl 
"En un sentido tanto inmediato como simbólico, tanto corporal como espiritual, somos a cada instante aquellos que separan lo ligado o ligan lo separado."

Georg Simmel, "Puente y puerta” (1909)

Este artículo se basa en algunas reflexiones surgidas a partir del trabajo de intervención arquitectónica de restauración de un inmueble de valor histórico -la casa Ehrenfeld, actual Conservatorio de Música de la Universidad Austral de Chile (UACh)-, en la ciudad de Valdivia ${ }^{4}$. Asimismo, ellas surgen de una labor de diálogo y observación entre dos disciplinas: la arquitectura y la antropología.

Entre los inmigrantes alemanes son algunas las familias que logran construir grandes casas, y la antigua calle de Los Canelos (actualmente, General Lagos) es escogida por varias de ellas. Algunas de esas casas tendrán sus instalaciones de producción en la Isla Teja y en lugares más extremos de la ciudad con acceso a la vía fluvial, como Miraflores, Las Ánimas y Collico. Las casas de industriales, como la Ehrenfeld, se construyen en los terrenos que se ubican entre esa calle y el río mismo. El apogeo industrial (Almonacid, 2013), que posibilita el desarrollo de una arquitectura y un urbanismo temprano de la antigua ciudadela colonial, convierte esta calle en una suerte de "barrio experimental" para esta burguesía, transformando al río en una amplia zona industrial y puerto fluvial, más amplio que la ciudad misma (Saelzer y Urbina, 2015). Lo que fue una creación particular, familia por familia, sitio a sitio, sumando instalaciones productivas y de transporte fluvial, adquirió en la totalidad de la calle una expresión colectiva urbana.

La exposición secuencial y documentación de los inmuebles de este barrio iniciada por el arquitecto y OSB Gabriel Guarda (1980) derivó en un interés colectivo por la herencia arquitectónica e histórica de estas casas, muchas de las cuales se habían deteriorado por consecuencia de la humedad y falta de mantenimiento, otras cuantas habían sido adaptadas como residencias institucionales, o bien, habían comenzado a desaparecer por demoliciones e incendios. Las diferentes formas de degradación han hecho difusos los valores materiales de este patrimonio y han dificultado el reconocimiento de los componentes culturales del barrio. Sin embargo, la singularidad de las casas y los primeros estudios de las mismas determinaron su valorización como barrio de inmuebles especiales. El conjunto quedó normado a nivel del Plan Regulador Comunal, en 1992, como "Zona típica", para permanencia conmemorativa de los inmuebles. 


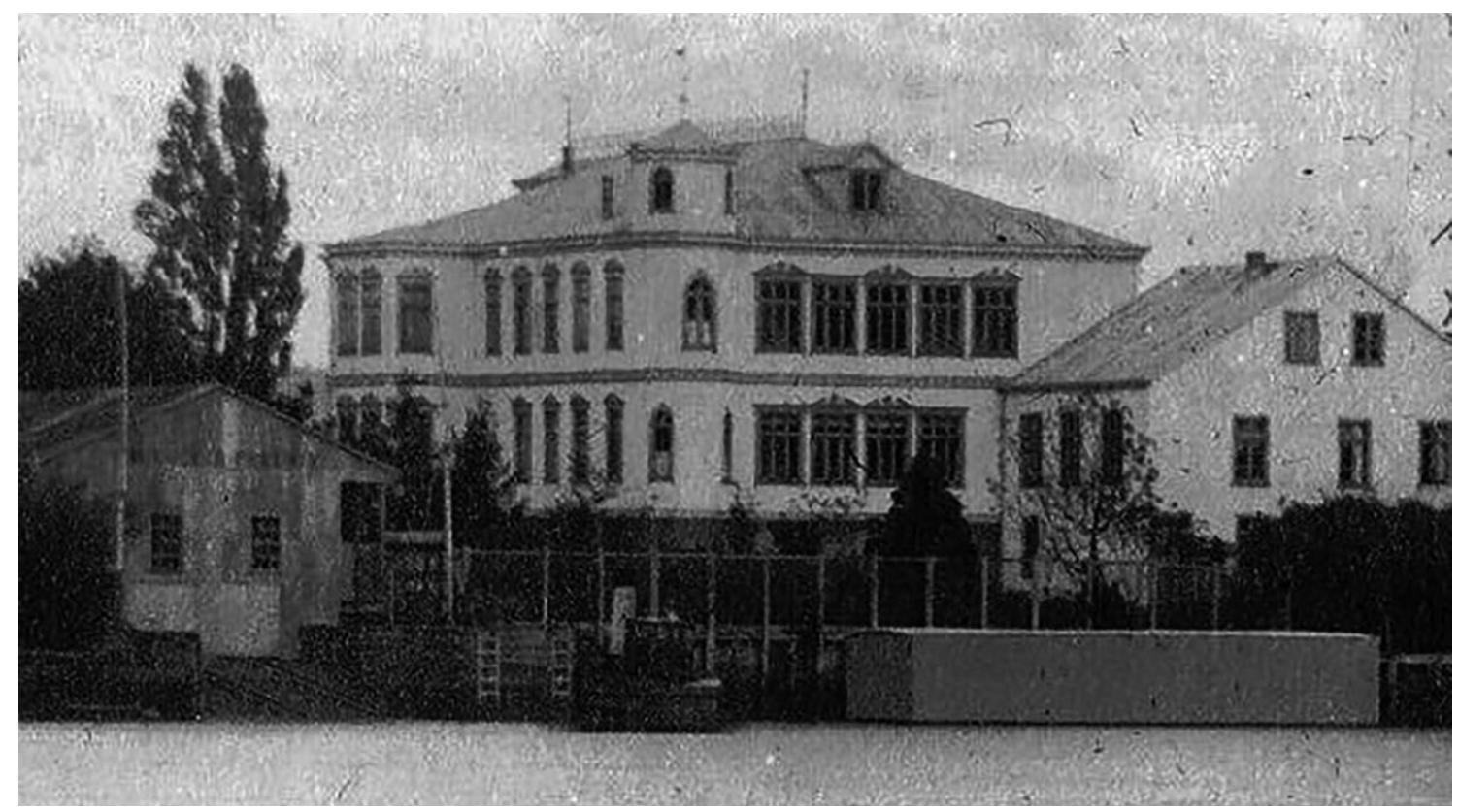

Figura 1 Casa Ehrenfeld, fachada norte y poniente ca., 1919. Fuente: Fotografía de Lorena Liewald.

En 1956 la casa fue adquirida por la Universidad Austral de Chile, siendo entonces utilizada para el funcionamiento de la Facultad de Bellas Artes, albergando actualmente al Conservatorio de Música. En la iniciativa acordada entre el Consejo de la Cultura y las Artes (CNCA) y la UACh, en 2013, mediada por los permisos otorgados por el Consejo de Monumentos Nacionales y la Dirección de Obras de la comuna, existe un consenso inicial: los inmuebles de valor histórico, urbano y cultural, como la casa Ehrenfeld (Zona Típica e Inmueble de Conservación Histórica) ${ }^{5}$, son entes portadores de una identidad que conjugan una cierta arquitectura y una determinada área urbana, y como tales incluyen información de una realidad que trasciende al solo edificio.

En este contexto, la singularidad de la restauración arquitectónica en la Zona Típica permite ampliar, si se quiere, la pregunta sobre el valor histórico de un inmueble. La experiencia de trabajar en estas edificaciones, crea la oportunidad de trasladar la respuesta de los instrumentos legales y fiscales hacia nuevas exploraciones sobre aquello que sustenta el trabajo "identitario" a nivel del patrimonio local $^{6}$. En este caso, se trata del hallazgo de espacios latentes en el inmueble y en el contexto que este genera, y de nuevas perspectivas de valoración cultural que podrían resultar relevantes para la comunidad.

\section{METODOLOGÍA}

Por el simple hecho de responder al encargo de restaurar un inmueble consignado por un valor histórico, el imaginario arquitectónico se ve enfrentado al "patrimonio", en su similar condición de imaginario: el de una casa antigua, de una historia local, urbana, por ejemplo. Este fue el punto de partida para intentar modalidades concretas y productivas del encuentro entre las maneras de trabajar (y de soñar) del antropólogo y del arquitecto. Tal como lo expresara el antropólogo francés, Alban Bensa, a propósito de su trabajo con Renzo Piano, "se trata, en el fondo, de dar a ver y, si es posible, a apreciar y a pensar una diferencia. El arquitecto lo expresa con líneas y materiales puestos en espacios; el antropólogo, con sus datos de campo puestos en texto. El uno y el otro lo hacen con su propio estilo, para dar cuerpo y vida a esta entidad misteriosa que llamamos cultura" (2000: 449).

Igualmente, se busca aquí aprender en términos de lo que el antropólogo Tim Ingold (2012) Ilama conocer desde dentro, intentando realizar un arte de la indagación que se mueve hacia adelante, en tiempo real, y uniéndose al movimiento del hacer arquitectónico, dialogando paso a paso, buscando una mirada. En la 


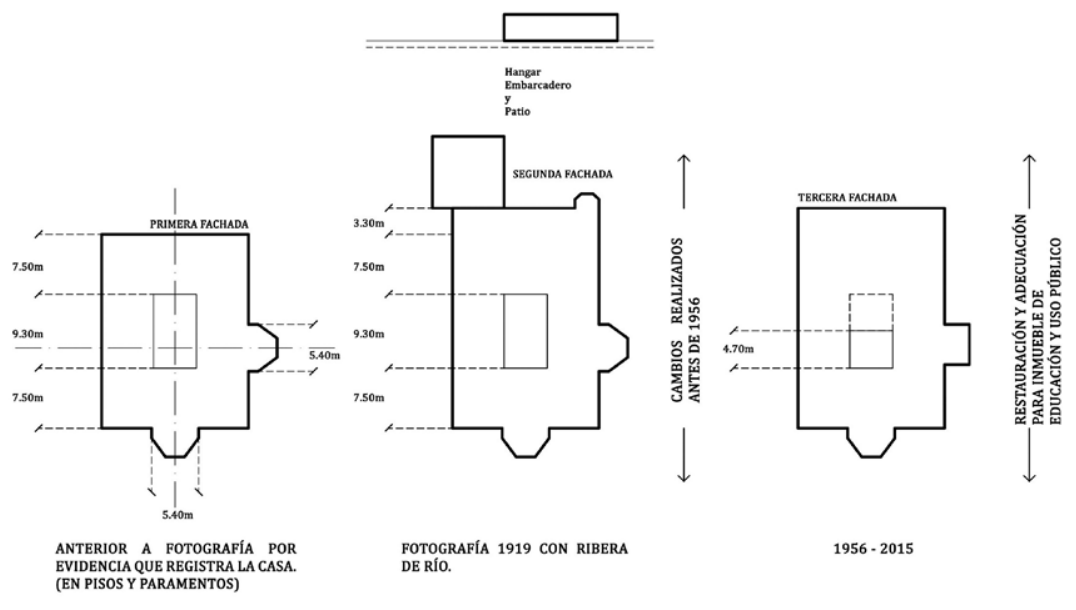

Figura 2 Caracterización histórico-arquitectónica en la planta de la casa Ehrenfeld. Fuente: Gerardo Saelzer

misma línea, el objetivo es también pensar a través del hacer (Ingold, 2013), mas no por un encuentro entre mentes ya equipadas de conceptos y un mundo material ya poblado de objetos, sino más bien por nuestros compromisos prácticos y observacionales con los materiales, los seres y los objetos que nos rodean durante/en los propios procesos del pensar.

El caso de la intervención en la casa Ehrenfeld permitió profundizar la idea de que las obras sobre las que recae la discusión de la restauración material ofrecen un campo que permite revisar las condiciones de posibilidad de cambios fisonómicos de la ciudad a través de los materiales y los actores, como la madera, en tanto materia prima, y la mano de obra adaptada a su elaboración mecánica desde la etapa colonial. Durante el trabajo de intervención arquitectónica, y de diálogo, fueron apareciendo formas de mirar y de considerar reflexivamente lo que iba surgiendo. Así, se repensó, por un lado, la concepción de "patrimonio" como un proceso cultural, ampliando con ello la mirada crítica, examinando sus consecuencias concretas, sopesando "la idea de que el patrimonio hace cosas, que tiene un efecto, que 'realiza un trabajo' social y cultural" (Smith, 2011: 41); y, por otro, la noción de "inmueble", como una suerte de nudo, un amarre de materiales que momentáneamente se mantienen unidos de manera coherente, considerando en este discurrir las historias de los materiales y de las vidas implicadas, pero también de otras hebras que conducen hacia otros lugares.

\section{RESULTADOS}

Para el equipo a cargo, el proyecto de intervención arquitectónica suponía una restauración de toda la casa, lo que permitía vislumbrar el comportamiento de una edificación completa y no solo de algunos de sus sectores. La casa Ehrenfeld presentaba un sinnúmero de alteraciones, muchas de ellas no registradas, mostrando con ello que un inmueble nunca se acaba del todo, que habitarlo supone siempre intervenirlo, puesto que, como lo declara el antropólogo Juan Carlos Olivares, "la arquitectura no muere a causa de la presencia y actividad del tiempo. Al contrario, ella es el tiempo" (2013: 26).

Debido a lo anterior, si bien el trabajo proyectado se pensó en base a cómo era la casa originalmente, a medida que éste avanzaba se descubrieron diversas modificaciones ${ }^{7}$ realizadas con posterioridad, lo cual limitó la posibilidad de dar plenamente con la estructura original. Varias son las alteraciones que imponen distancias entre el inmueble construido y el inmueble habitado en la actualidad. Lo que se tiene, entonces, es el encuentro con elementos de una casa formada por distintos tiempos. Debido a ello, el procedimiento de intervención se debate teóricamente entre dos posibilidades: la reconstrucción y/o la puesta en valor del inmueble (que en parte coinciden y en parte divergen). Ambos lineamientos quedan tensionados cuando responden a las políticas públicas y al financiamiento de intervenciones arquitectónicas patrimoniales para un uso público. 


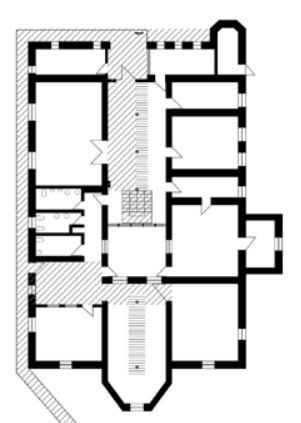

PLANTA PISO ZOCAL
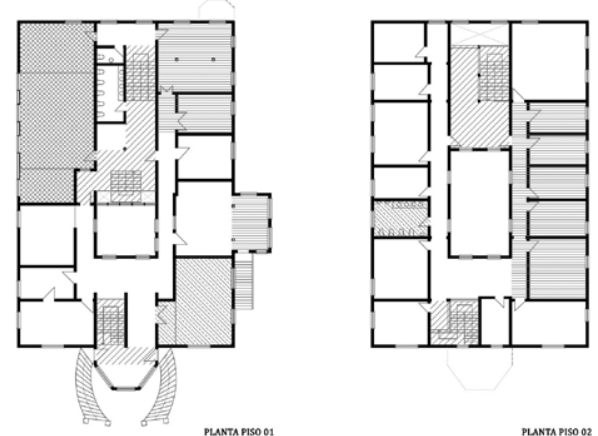

A 1 Adecuación a funciones públicas incorporadas entre 1956 y 2015.

B Adecuacín a funciones públicas y servicios 2015 - 2016 .

c WC Cumplimiento de normativas de desplazamiento y seguridad

D E Refuerzo estructural y reconstruccion por dafios profundos.

Figura 3 Intervención de la Casa Ehrenfeld para su uso público. Fuente: Gerardo Saelzer

ELEMENTOS EN LA PLANTA 1956 - 2015 QUE VISLUMBRAN INTERVENCIONES ANTERIORES Y EL ESTADO EN 1919.

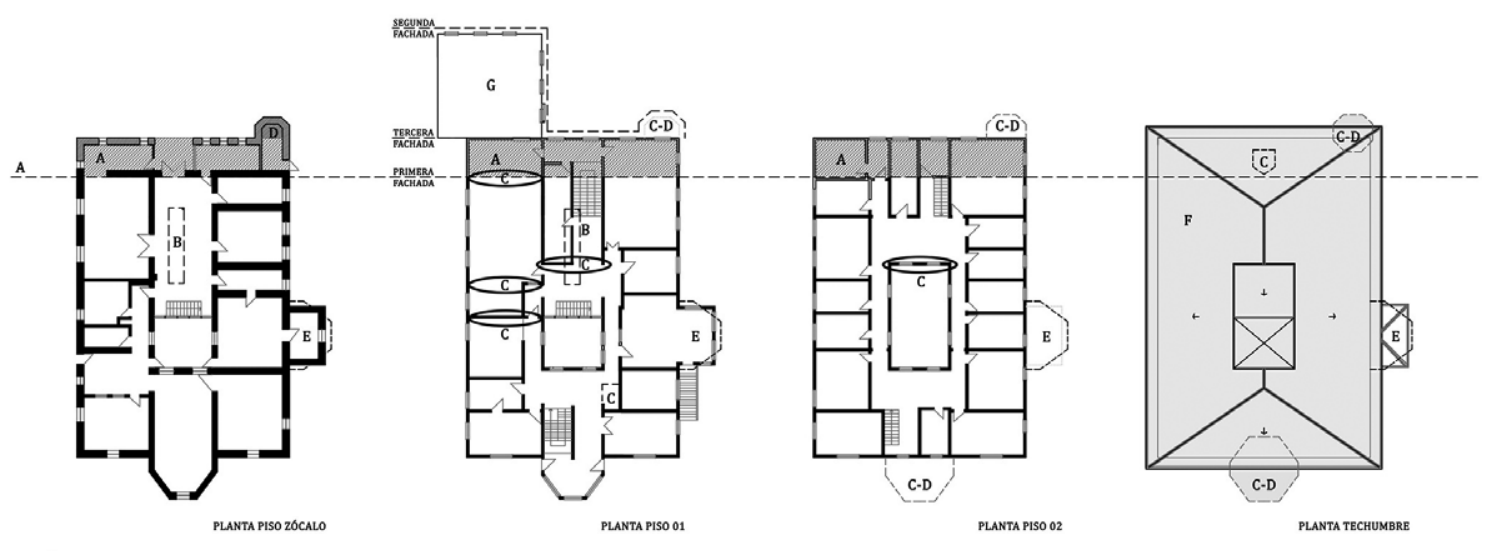

A Área que denota una edificació adosada.

Evidencias de estructura descontinuada que denotan la eliminación de paramento, recintos y otras intervenciones

Base de la torre de cuatro pisos evidenciada en la fotogralla

Modificación de saliente pentagonal, de tres pisos, hoy rectangular y dos pisos

Volumen sur-poniente solo atestiguado en la fotografia

Figura 4 Elementos en la planta, 1956 - 2015. Fuente: Gerardo Saelzer 
Se constató que el presente acumula las marcas sobre la edificación original: estructuras que indican los cambios, deterioro, desaplomes, materialidades de las alteraciones, lo que constituye la herencia del pasado en el presente. Se trataba, también, de operar un rescate antes de que el edificio colapsara. Incluir el paso del tiempo, sus marcas, al restaurar, conllevaba discriminar etapas de intervención y discriminar también entre los elementos originales y exógenos.

El valor patrimonial oficial de la casa Ehrenfeld recae en el cuerpo arquitectónico, presentado en sus aspectos exteriores. Sin embargo, ello invisibiliza la obsolescencia de los usos originales del inmueble, la dinámica social de la que se discute y la pérdida de elementos constitutivos, como jardines y uso del borde fluvial, algunos irrecuperables de no mediar un sinnúmero de voluntades.

\section{Tres tipologías}

El concepto de "inmueble de conservación histórica" integra aspectos materiales e inmateriales con que, en parte, se ha venido analizando la noción de patrimonio: el inmueble propiamente tal, en cuanto edificación, desde su concepción original hasta el estatus actual; y el valor histórico, en tanto que marco temporal abordado críticamente y el reconocimiento de una existencia que nos antecede y que se relaciona con el destino urbano. Para constituir conceptualmente el contexto generado por estas edificaciones, se hizo necesaria una metodología de caracterización espacial y temporal de los inmuebles constitutivos del valor histórico en/de la Zona Típica.

Las tipologías arquitectónicas suponen condiciones estructurales, por lo que su debate conceptual conlleva contemplar cambios históricos y paisajísticos de la fisonomía urbana (cf. Mallgrave, 2009). En este caso particular, ello involucraba una primera organización de características arquitectónicas, así como de la (posible) evolución histórica de este sector urbano. De esa forma, se conjeturaba una práctica y una producción de diversos modelos arquitectónicos que obedecerían a patrones de edificación y a ciertas variantes culturales. Es así como estas casas, como casos de adaptación de modelos arquitectónicos centroeuropeos, se sitúan entre conocimientos extranjeros y otros conocimientos locales, como aquellos relacionados con la disciplina, la elaboración y el trabajo metodológico con la madera (Cherubini, 2016; Guarda, 2001)

Con todo, se establecieron tres estados: (1) la edificación temprana de los inmigrantes alemanes, cuando éstos se debatían entre el proceso de asentamiento en calidad de "artesanos" y la incorporación de maquinaria, que generaría dependencia de ella como también nuevos procesos de producción, como sucede con las mueblerías con taller (Almonacid, 2013: 41); (2) la edificación como resultado del éxito económico de estos inmigrantes alemanes: casas con sentido aristocrático, familias que transmiten un sentido civilizatorio en la ciudad (3) la edificación que responde a la calle como espacio que valida a la arquitectura, relegando el área verde del predio al interior de la manzana o al patio de un conjunto de viviendas.

En la Zona Típica, donde surgen estas observaciones, el primer grupo lo denominamos "tipo rural"; "tipo villa" al segundo, y "tipo urbano" al tercero.

Las primeras soluciones formales, de tipo rural, tienen como referente a aquellas que se encuentran con regularidad en asentamientos del norte de Europa, identificadas en los registros fotográficos sobre Valdivia, que dan cuenta de un recambio del casco coIonial histórico (Saelzer y Urbina, 2015). Las segundas soluciones responden a la concepción de la arquitectura de las "villas", modelos únicos o exclusivos, cuya relación naturaleza-arquitectura establece nuevos valores estéticos y urbanos que modifican la ciudad (cf. Boucher, 1998). Adquiere importancia la composición de fachadas y la ornamentación como estrategia para proponer lo distintivo: una naturaleza que penetra en la arquitectura (sino en todas, en tres de las fachadas), acompañada además por un cierro ornamental de hormigón y acero forjado, que reemplazaría al cierro de las casas tipo rural, hecho de malla de acero trenzado sujeto por delgados marcos de acero (es el caso de la casa Ehrenfeld). Las terceras soluciones re-elaboran modelos arquitectónicos ${ }^{8}$ que abarcan desde 1854 hasta el s. XX, en la descripción de Guarda: "La casa mantiene la planta concentrada, la doble crujía y el patio adicional con piezas de servicio; desaparece el comedor exterior, la gran herencia española, odiada por Pérez Rosales: 'deseando dar a esta población un aspecto europeo' " (2001: 572).

Hasta ahora la historiografía urbana de Valdivia se ha basado en definiciones generales como "sustitución (de casas) hechas por constructores alemanes", "profesionales y eximios artesanos", que "renuevan la fisonomía urbana", "revolución arquitectónica" y "nuevas técnicas" que en "breve plazo" levantan "elegantes construcciones de última moda", "moda neoclásica", "aspecto edilicio" (ibídem, 570-573). Sin embargo, lo que se observa, a medida que estos inmuebles se van construyendo, es algo más. Se puede conjeturar aspectos demográficos; deseos de realizar negocios de arriendo; reubicación de familias de inmigrantes en la escala económica o social; problemáticas de edificación; incluso, sintonía con la formación nacional de Alemania ligada a la arquitectura que la caracteriza en el desarrollo de las ciudades, como la dicotomía histórica arquitectura - ingeniería que lleva a preguntarse con qué formación (Ausbildung) contaban los artesanos de origen alemán avecindados en la zona, como lo discute Almonacid (2013: 84-87) y Cherubini (2016: 37-46). Lo que sí es posible asegurar es que la estructura social ya está en movimiento durante el período de edificación de los inmuebles, hoy históricamente valorizados, y esos procesos conectarían a Valdivia con otros procesos dinámicos contemporáneos suyos. 

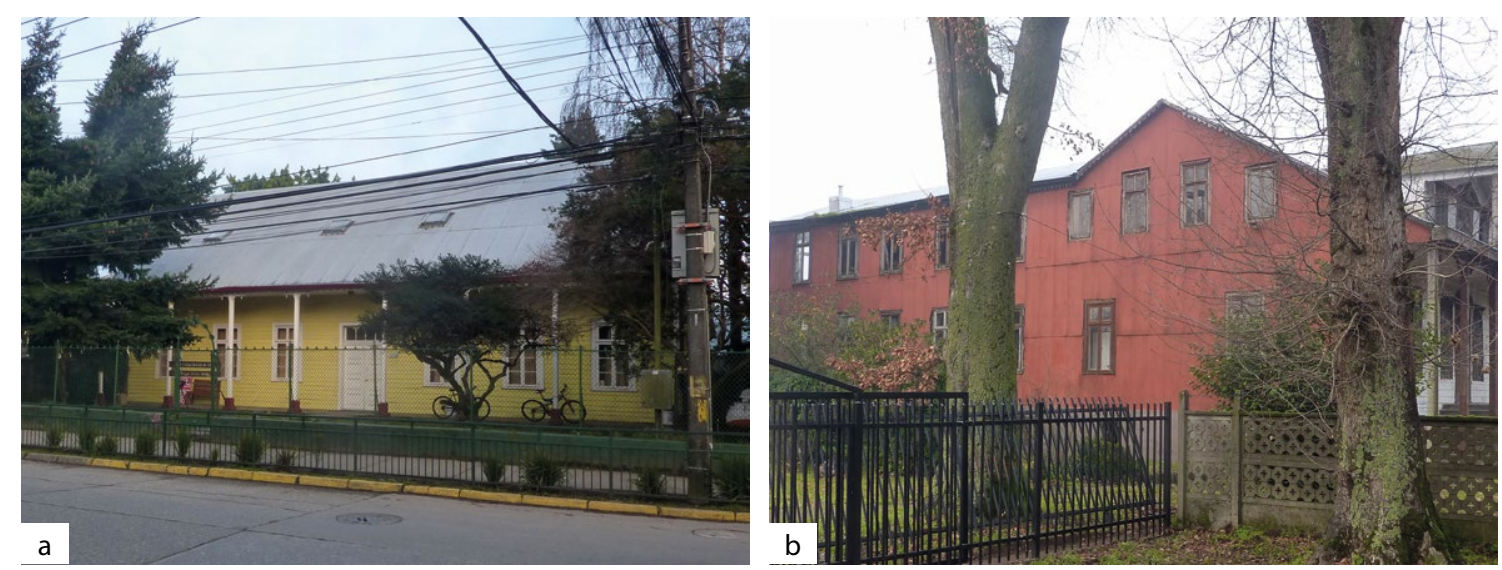

Figura 5 (a) Tipología rural. General Lagos 1101, actual Hogar estudiantil UACh. Fuente: Fotografía de Gerardo Saelzer. (b) Tipología rural. Casa Haverbeck. Fuente: Fotografía de Gerardo Saelzer
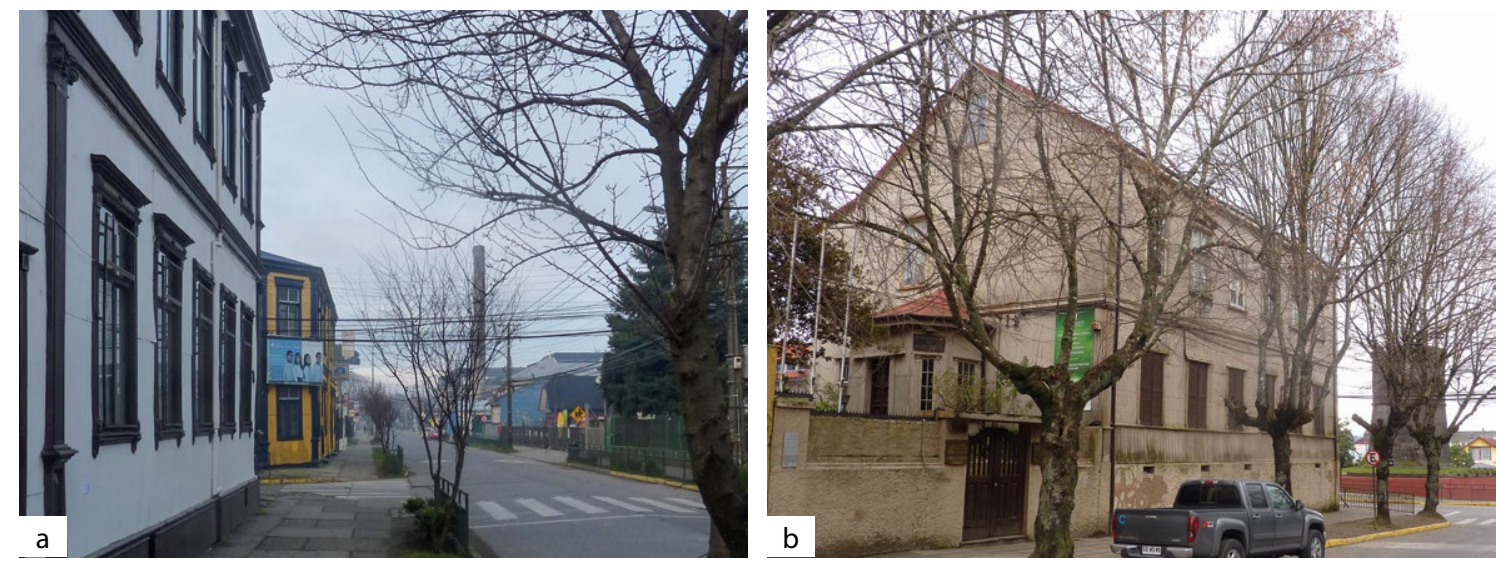

Figura 6 (a) Tipología urbana. Casas Paulsenberger. Fuente: Fotografía de Gerardo Saelzer. (b) Tipología urbana. Casa HoffmannDeppe, actual Luis Oyarzún - UACh. Fuente: Fotografía de Gerardo Saelzer
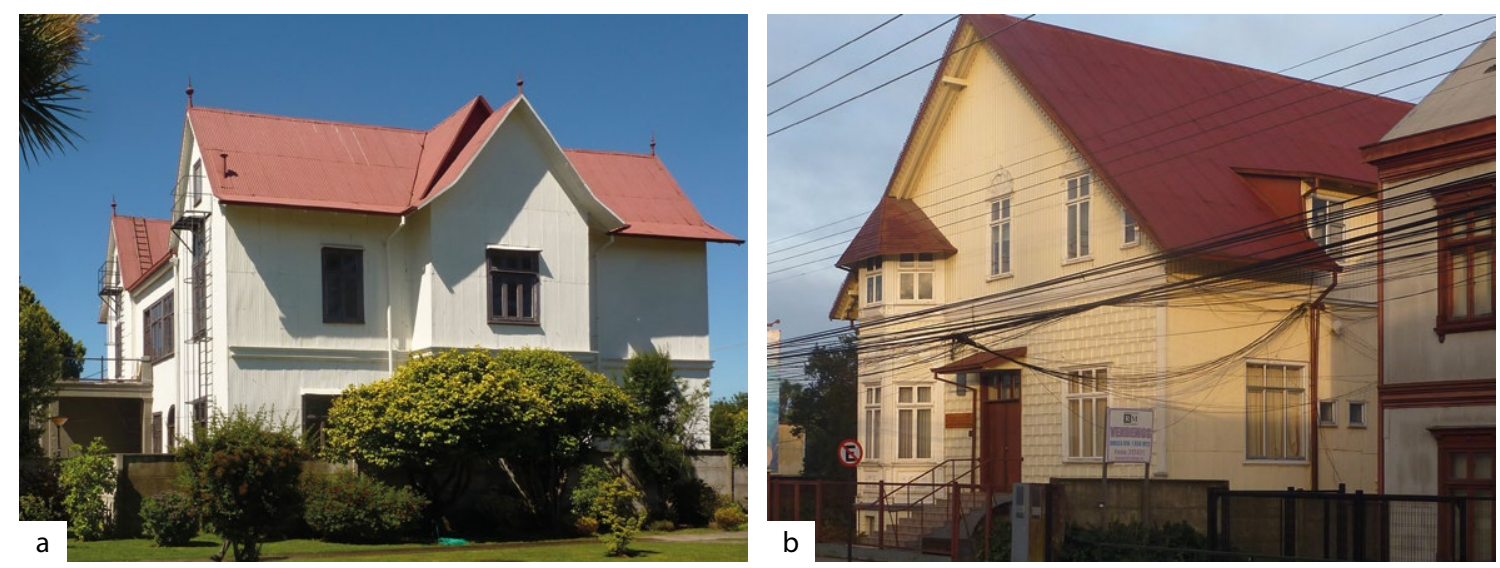

Figura 7 (a) Tipología villa. Casa Commentz-Hoffmann. Fuente: Fotografía de Gerardo Saelzer. (b) Tipología villa. Casa Stillfried. Fuente: Fotografía de Gerardo Saelzer 

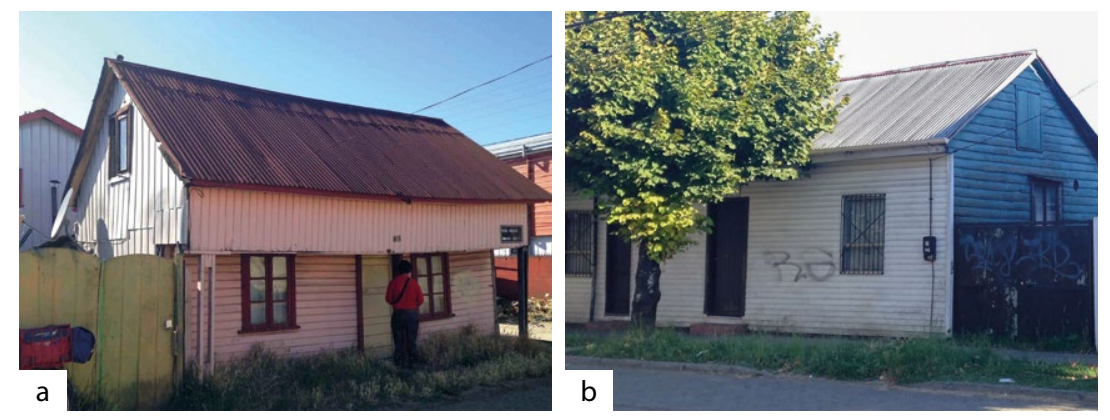

Figura 8 (a) Tipo rural. Pérez Rosales 1676. Fuente: Fotografía de Gerardo Saelzer. (b) Tipo rural. Bueras 850 Fuente: Fotografía de Gerardo Saelzer
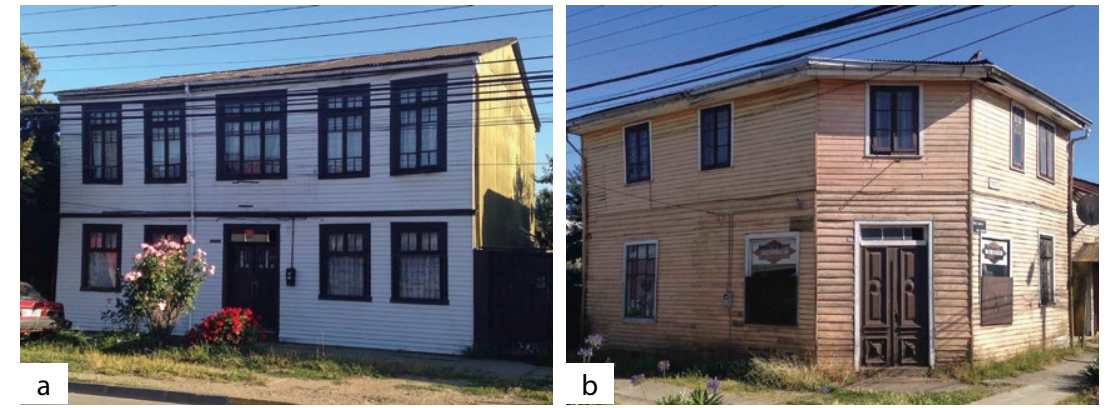

Figura 9 (a) Tipo urbana. Bueras 352. Fuente: Fotografía de Gerardo Saelzer. (b) Tipo urbana. Pérez Rosales 192 esq. Domeyko. Fuente: Fotografía de Gerardo Saelzer
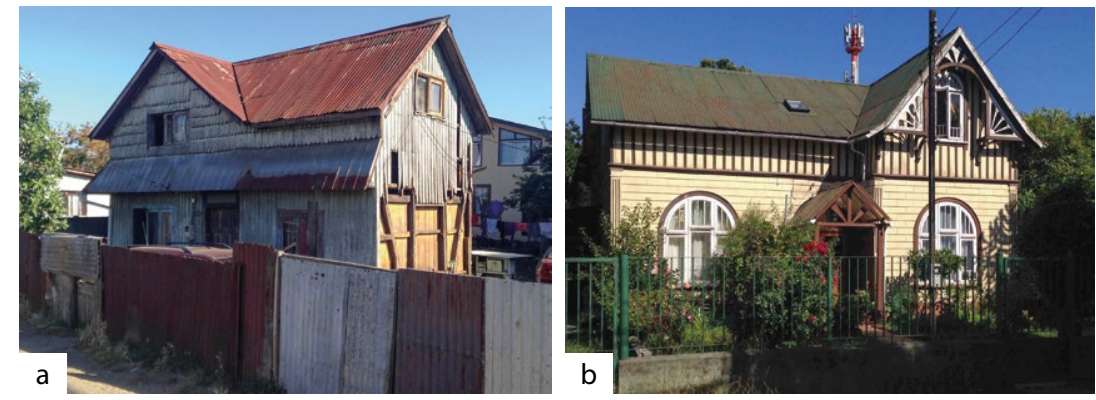

Figura 10 (a) Tipo villa. Pasaje Haverbeck s/n. Fuente: Fotografía de Gerardo Saelzer. (b) Tipo villa. Pérez Rosales 1445. Fuente: Fotografía de Gerardo Saelzer
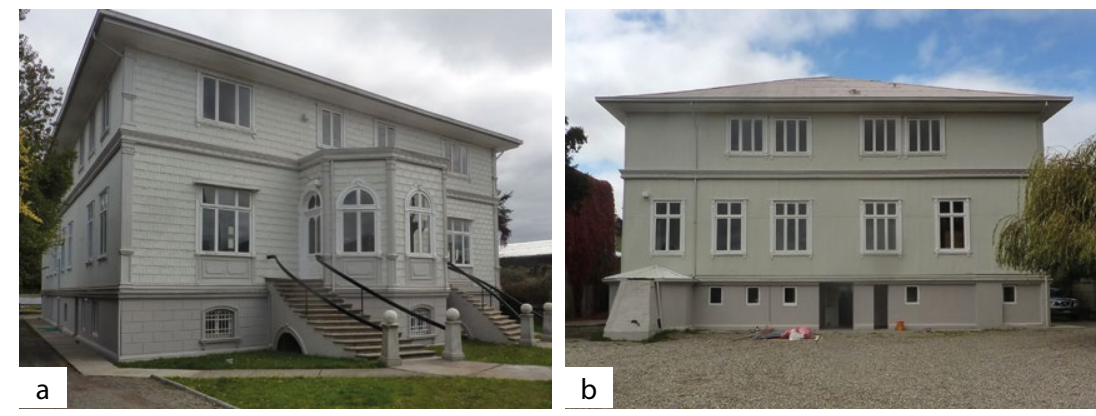

Figura 11 (a) Casa Ehrenfeld (tipo villa), fachada oriente, 2016. Fuente: Fotografía de Gerardo Saelzer. (b) Casa Ehrenfeld (tipo villa), fachada poniente, 2016 Fuente: Fotografía de Gerardo Saelzer
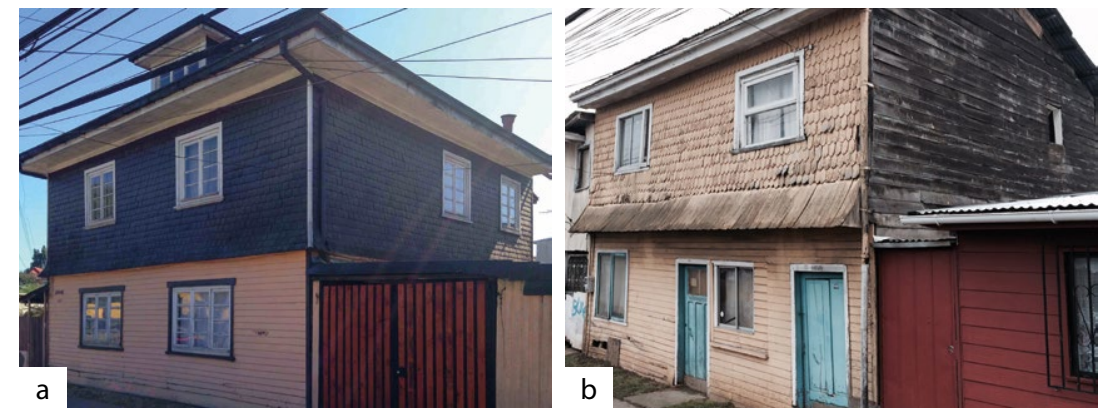

Figura 12 (a) Casa tipo villaurbano. Sotomayor 1048 Fuente: Fotografía de Gerardo Saelzer. (b) Casa tipo villa urbano. Clemente Escobar 980 Fuente: Fotografía de Gerardo Saelzer 


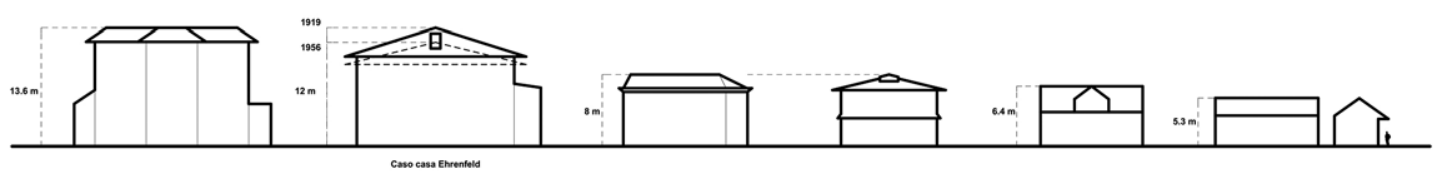

Figura 13 Composición de alturas a partir de la tipología de villa. Fuente: Gerardo Saelzer

La concreción de los encargos de construcción, basados estos en las concepciones sociales y urbanas de una generación que deja atrás la condición de inmigrantes y se asienta como núcleo cultural, es posible gracias a funciones y a imaginarios arquitectónicos que contienen citas a "diseños" e "ingenieros o constructores". Esas huellas permiten caracterizar tanto a los clientes como a los constructores que formaron la primera generación de procesos constructivos adaptados a las condiciones locales.

Si en esta "primera generación", los talleres actuaron sobre la base de la práctica profesional y la referencia teórica a modelos arquitectónicos centro-europeos, las ejecuciones de la segunda y sucesivas generaciones fueron surgiendo como práctica artesanal de "saberes adquiridos" a partir de las edificaciones primeras.

\section{Barrios e interior: la ciudad de otra manera}

Recorriendo las actuales calles Yungay y General Lagos, y los barrios que se formaron junto a ese eje, se observa que los inmuebles arquitectónicos consignados como patrimonio son un aspecto inicial de saberes materializados. Edificaciones como la casa Ehrenfeld no bastan para dar respuesta al escenario producido, y parcialmente heredado, que fue dando forma a la ciudad de Valdivia. Debido a que tales edificaciones permiten vincular la interpretación histórica a un proceso de formación de la ciudad, a barrios de madera, modos de existencia y formas de vida, el estudio condujo a la observación de los barrios obreros formados al costado del eje General Lagos para examinar visualmente sus urbanizaciones.
Lo que nació a orillas del río (edificaciones industriales y viviendas de una clase acomodada) se diseminó al interior de la ciudad. Se trata de apropiaciones de tipologías arquitectónicas, de estructuraciones de prácticas situadas, que se originan en los actores y las materialidades. Similitudes entre casas de variados tamaños, en diversos lugares de las cuadras cercanas, construidas en el período que atañe al patrimonio que aquí interesa, permite suponer que la mano de obra, especialmente carpinteros, trasladaron conocimientos y práctica arquitectónicos fuera del núcleo de la "primera generación" hacia otros sectores de la ciudad. Es así como se habrían generado procesos de apropiación y de diseminación de las prácticas, especialmente de carpintería. Conocimientos y práctica arquitectónicos de la "segunda generación" se caracterizan como réplica de cierta influencia del trabajo manual así como también de la fisonomía de las casas. Devenidas en prácticas artesanales obreras, moldearon inmuebles concebidos fuera de la calle de Los Canelos y sus equivalentes en otros sectores de la ribera en Valdivia.

Lo que se advierte, entonces, son otras experiencias estéticas llevadas a cabo en aquellas casas, reproducidas en edificaciones bajo, posiblemente, otros cánones de encargo. Es decir, un trasvasije artesanal de aquella arquitectura ejercitada en tales casonas hacia arquitecturas que, aun manteniendo la fisonomía, han aplicado composiciones que se alejan de tales edificaciones; por ejemplo, tipología de villas mediante transformaciones que modificaron las escalas, los ventanales, las empequeñecieron, eliminaron el zócalo, el antejardín, entre otros. 


\section{CONCLUSIONES}

Las condiciones actuales para la recuperación de las edificaciones que conforman lo que denominamos el caso patrimonial de esta Zona Típica, comprenden, naturalmente, las condiciones históricas, legales y técnicas que gravitan sobre el barrio. Los procesos dinámicos responden a la integración de aspectos materiales e inmateriales con que se ha venido discutiendo la noción de patrimonio. Estas dinámicas, así como las construcciones, ofrecen campos de exploración basados en los actores de los procesos de edificación y su influencia en otros lugares de la ciudad. Las condiciones legales, si bien convocan los esfuerzos en una zona, no completan el mosaico urbano. Lo anterior es verificado por la evidencia empírica que representan, en primer lugar, los modelos de viviendas edificadas detrás de la Zona Típica, conjunto denominado "Barrios bajos"; evidencia que se extiende, en los mismos años, al casco original de la ciudad y a nuevos barrios, hoy en discusión como patrimonio de adaptaciones locales (Ojeda, 2014).

Convengamos en que el espacio vivido -el de los habitantes y usuarios-diverge del espacio concebido -el del planificador, el arquitecto y la arquitectura (Delgado, 1999). Tras ese espacio concebido y representado hay ideología: un fantasma que recubre las relaciones sociales reales de producción. De ello debe tener conciencia toda intervención arquitectónica. Nos parece que en el caso de Valdivia, el peligro yace en vincular el valor histórico de los inmuebles habitacionales con un tipo de burguesía. La solución propuesta se basa en el gesto de interpretar el valor histórico: inmuebles detonantes de cambios en la ciudad, creación de fisonomía de esos y otros barrios. Se trata de procesos que derivan desde el espacio concebido al espacio vivido. La ciudad, en este caso, aparece como un medio en el que las identidades se dejan leer en la superficie. Y, como nos lo recuerda Isaac Joseph, "la superficie como lugar del sentido es precisamente la experiencia antropológica del presente que vaga por la ciudad (1988: 48).

La necesidad de restaurar, de intervenir la casa Ehrenfeld, sirvió de excusa para reformularla y proponer con ello al menos una mirada sobre un barrio de valor histórico, un contexto, una ciudad. Lo que la presente experiencia deja vislumbrar es que una colección de inmuebles en un espacio común y una re-interpretación de los mismos, a modo de eco, en otros sectores de la ciudad, abre nuevos espacios de interpretación. De esta manera, "la ciudad se vuelve densa al cargarse con fantasías heterogéneas. La urbe programada para funcionar, diseñada en cuadrícula, se desborda y se multiplica en ficciones individuales y colectivas" (García-Canclini, 2000: 109).

Estas intervenciones permiten comenzar a discutir temas importantes de la sociedad, sus ciudades y territorios ${ }^{10}$. En ese tenor, también habrá que decidir si asumir o dejar en suspenso, mantenidas a raya, las dinámicas sociales que impregnan y recorren todo espacio, que lo construyen en su actual inestabilidad y en su inestable actualidad (Araya, 2015). Se necesita, en definitiva, avanzar desde la descripción del caso paradigmático que tradicionalmente ha marcado nuestra mirada sobre el espacio y el tejido urbano, hacia los procesos. Esto alude no solo a la producción de conocimiento, sino también a una decisión política. Pues, tal como lo plantean los arquitectos Josep Maria Montaner y Zaida Muxí: "La primera decisión política -en cualquier actividad de teoría, historia y crítica del arte y la arquitectura-radica en lo que se visibiliza y en lo que se oculta, en lo que se dice y en lo que se calla y a quién se silencia" (2011: 16). 


\section{REFERENCIAS BIBLIOGRÁFICAS}

ALMONACID, Fabián. La industria valdiviana en su apogeo (1870-1914). Valdivia: Ediciones Universidad Austral de Chile/Kultrún, 2013.

ARAYA, Pedro. Lo que aparece, lo que queda. En: Castillo Gómez, Antonio (ed.), Culturas del escrito. Del Renacimiento a la contemporaneidad. Madrid: Casa de Velázquez, 2015, pp. 45-63.

BENSA, Alban. L'ethnologue et l'architecte. La construction du Centre Culturel Tjibaou. Revue de synthèse, julio-diciembre 2000, no 3-4, pp. 437-451.

BOUCHER, Bruce. Andrea Palladio: the architecture in his time. Nueva York: New York Abbeville Publishing, Library of Congress Catalog, 1998.

CNCA. Plan Nacional del Fomento a la Economía Creativa. Santiago: Consejo Nacional de la Cultura y las Artes, 2017.

CHERUBINI, Gian Piero. La Escuela de Carpinteros Alemanes de Puerto Montt. Santiago: Editorial Universitaria, 2016.

DELGADO, Manuel. El animal público. Pasos hacia una antropología de las calles. Barcelona: Anagrama, 1999.

DELGADO, Manuel. La ciudad mentirosa. Fraude y miseria del "modelo Barcelona". Madrid: La Catarata, 2007.

GARCÍA-CANCLINI, Néstor. Imaginarios urbanos. Buenos Aires: Eudeba, 2000.

GÓMEZ IBÁÑEZ, José y MCCUE, Gerald M. (eds.). Revitalización del casco histórico de Toledo: estudio de estudiantes y profesores de Harvard. Minneapolis: Bolger Publications, 1995.

GUARDA, Gabriel. Conjuntos urbanos histórico-arquitectónicos. Valdivia, s. XIX-XX. Santiago: Ediciones Nueva Universidad, 1980.

GUARDA, Gabriel. Nueva Historia de Valdivia. Santiago: Ediciones Pontificia Universidad Católica de Chile, 2001.

INGOLD, Tim. Conociendo desde dentro: reconfigurando las relaciones entre la antropología y la etnografía [en línea]. Conferencia pronunciada en la Universidad Nacional de General San Martín, 2012. [Consultado 13 abril 2017]. Disponible en: www.unsam.edu.ar/ antropologia/wp-content/uploads/2012/11/Conferencia_Ingold_UNSAM.pdf

INGOLD, Tim. Making: Anthropology, Archaeology, Art and Architecture. Londres: Routledge, 2013.

JOSEPH, Isaac. El transeúnte y el espacio urbano. Ensayo sobre la dispersión del espacio público. Barcelona: Gedisa, 1988.

MALLGRAVE, Harry Francis. Modern Architectural Theory: a Historical Survey, 1673-1968. Cambridge Ma.: Cambridge University Press, 2009.
MONTANER, Josep Maria y MUXÍ, Zaida. Arquitectura y política. Ensayos para mundos alternativos. Barcelona: Ed. Gustavo Gili, 2011.

OJEDA, Pablo. Casa Barrios Bajos, Valdivia, Chile, 20132014. Revista $A R Q$, diciembre 2014, n88, pp. 86-87.

OLIVARES, Juan Carlos. Rupameika blues / etnografía de la arquitectura Mapuche Williche de la Puel Mapu. En: CORDERO, Elisa (ed.). Taller [Sur] 2012. Patrimonio Cultural sostenible. Visiones, prácticas e innovación desde la arquitectura. Valdivia: Instituto de Arquitectura y Urbanismo, UACh, 2013, pp. 16-26.

PAHL, Burkhard; HOBUSCH, Tom; KRUGER, Sabine y PLUTO, Christine. Neue Wohnkonzepte im Gründerzeitbestand [en línea]. Stuttgart: Fraunhofer IRB Verlag, 2003. [Consultado 13 abril 2017]. Disponible en: https://www.irbnet.de/daten/kbf/kbf_d_F_2443.pdf

SAELZER, Gerardo. Casa Ehrenfeld/Conservatorio de Música. Proyecto de intervención patrimonial. Valdivia: Universidad Austral de Chile, 2016.

SAELZER, Gerardo y URBINA, Simón. Urbanismo fluvial en el apogeo industrial de Valdivia: desaparición y recuperación (ca. 1850-2012), Revista Urbanismo, FAU Universidad de Chile, 2015, pp. 97-123.

SMITH, Laurajane. El 'espejo patrimonial'. ¿llusión narcisista o reflexiones múltiples?, Antípoda $\mathrm{n}^{\circ} 12$, enerojunio 2011, pp. 39-63. 